\title{
Anabases
}

ANABASES Traditions et réceptions de l'Antiquité

17 | 2013

Varia

\section{David Scott WILSON-OKAMURA, Virgil in the Renaissance}

\section{Amedeo Alessandro Raschieri}

\section{OpenEdition \\ Journals}

\section{Edizione digitale}

URL: http://journals.openedition.org/anabases/4298

DOI: 10.4000/anabases.4298

ISSN: 2256-9421

\section{Editore}

E.R.A.S.M.E.

\section{Edizione cartacea}

Data di pubblicazione: 1 marzo 2013

Paginazione: 308-309

ISSN: 1774-4296

\section{Notizia bibliografica digitale}

Amedeo Alessandro Raschieri, "David Scott wilson-okamura, Virgil in the Renaissance ", Anabases

[Online], 17 | 2013, Messo online il 01 avril 2013, consultato il 22 septembre 2020. URL : http://

journals.openedition.org/anabases/4298; DOI : https://doi.org/10.4000/anabases.4298

Questo documento è stato generato automaticamente il 22 settembre 2020.

(c) Anabases 


\title{
David Scott WILSON-OKAMURA, Virgil in the Renaissance
}

\author{
Amedeo Alessandro Raschieri
}

\section{NOTIZIA}

David Scott WILSON-OKAMURA, Virgil in the Renaissance, Cambridge-New York, Cambridge

University Press, 2010, $299+$ xiii p.

61 livres / isbn 978-0-521-19812-7.

1 Negli ultimi anni la fortuna di Virgilio durante il Rinascimento è stata oggetto di numerosi studi e convegni; basti ricordare l'incontro organizzato a Mantova nell'ottobre 2012. Wilson-Okamura in tale panorama utilizza una metodologia originale che parte dall'analisi dei più diffusi commenti rinascimentali all'opera virgiliana e dalle imitazioni dei maggiori poeti coevi, letti nelle edizioni cinquecentesche. La ricerca procede con la selezione di temi significativi, così da evitare una dispersione dell'argomentazione senza la pretesa di un'irraggiungibile esaustività. Se un pregio della ricerca risulta la sottolineatura degli elementi di continuità nelle trattazioni su Virgilio in età antica, medioevale e umanistica, risulta però eccessiva l'attenzione al mondo inglese, nonostante la consapevolezza del suo ritardo rispetto al resto dell'Europa.

2 Il libro, oltre all'introduzione, si sviluppa di tre parti, suddivise in sei capitoli. La prima sezione (Publication) consta di un solo capitolo (Virgil with an i), basato sull'analisi statistica del mercato editoriale virgiliano in età rinascimentale (le relative tabelle sono raccolte nelle appendici al volume). Per esemplificare la diffusione limitata delle innovazioni nella critica del Cinquecento è seguito il caso della grafia alternativa Virgilius/Vergilius, con le corrette argomentazioni di Poliziano, osteggiate o, almeno sottovalutate, da altri studiosi. Inoltre, è sottolineato il fatto che, insieme a una certa inerzia del pensiero, la maggior diffusione di alcune idee è legata alla forma editoriale: 
sono più volte stampati e diffusi i commenti puntuali, meno originali ma più utili a studenti e docenti nella quotidiana pratica didattica.

3 La seconda sezione del volume inizia con un capitolo dedicato all'opera pastorale di Virgilio (Patronage and the Eclogues). Per influenza del cosiddetto Donatus auctus, la vita del poeta è considerata come "a tale of patronage» nelle sue relazioni con il principe, così da diventare un modello per il cortigiano rinascimentale. Dal punto di vista del generale, questa parte della produzione virgiliana è avvicinata al genere dell'encomio, che si presta talvolta ad accuse di piaggeria. Nei commenti sono quindi evitati punti oscuri, come le critiche al sovrano, mentre sono valorizzate l'erudizione e la letterarietà, con l'imitazione di Teocrito. Altri aspetti importanti sono ritenuti l'amore e i legami con la profezia cristiana e l'epicureismo, mentre, dal punto di vista stilistico, è apprezzata la varietà e l'utilizzo del sermo humilis.

4 Come mostrato nel capitolo seguente (Variety and the Georgics), i commentatori rinascimentali, se certo riconobbero le caratteristiche di varietà, manifestata nell'erudizione e nella versatilità, di un poeta dotto capace di spaziare tra letteratura e filosofia, ebbero una certa difficoltà a classificare le Georgiche dal punto di vista dei generi letterari. In una visione dinamica di contenuto e forma, grazie alla ripresa di teorie medievali (la rota Virgilii), la produzione virgiliana così fu sistemata in uno schema organizzato per generi e stili. In particolare, il poeta antico diventa l'imitatore per eccellenza della natura, a sua volta imitato dai poeti rinascimentali: «Once the imitator, now the imitated, Virgil is the new and improved Nature» (p. 99).

5 La sezione si chiude con un capitolo su Morals and Minimalism, in cui è evidenziata soprattutto l'immagine del poeta come specialista del labor limae, come un perfezionista della parola, contraddistinto dalla purezza stilistica (così da diventare il modello ottimale per gli autori contemporanei) e dalla purezza morale, conseguente al suo pudore nel trattare argomenti erotici. In tale quadro, sono sterilizzate le dicerie già antiche sulle sue preferenze sessuali, la questione dell'omosessualità, manifestata nelle figure di Eurialo e Niso, o la presenza di Ecloghe pederotiche, che diventano nei commenti rinascimentali esempi di amicizia, amore platonico o semplici interventi di falsifica-zione. Virgilio è considerato il poeta perfetto, anche nei confronti dei suoi modelli: Ennio è superato per lo stile, Omero per brevità ed eloquenza.

6 L'ultima parte del volume è dedicata all'Eneide e intende offrire uno sguardo d'insieme sulle interpretazioni del poema tra 1300 e 1600, a partire dal diverso atteggiamento nei confronti delle due parti in cui si divide l'opera (libri 1-6, 7-12). Il primo capitolo (Virgil's Odyssey), oltre a mostrare come la caduta di Troia sia stata intesa come la fine del dominio dei sensi, evidenzia che l'argomento più commentato è la sezione sull'Aldilà, considerata una visione attraverso il sogno, nei suoi possibili legami con la necromanzia, ma anche passibile di interpretazioni allegoriche sul tema della ricchezza o della discesa verso la corruzione. Non mancano interpretazioni cristiane della reincarnazione, letta alla luce della dottrina sul purgatorio, che tuttavia costituiva un problema teologico presso le chiese riformate, o riflessioni politiche legate al rapporto col potere come emerge nella profezia dinastica.

7 Nell'ultimo capitolo (Virgil's Iliad) l'autore mostra che non è possibile individuare un episodio dominante nei commenti alla seconda parte dell'Eneide. Se da un lato Turno è visto come un eroe tragico, dall'altro si pone il problema del finale, soprattutto per la definizione di pietas in correlazione alla compassione, alla necessità della vendetta e all'ira di Enea. Sull'interpretazione del poema si riflettono: la contemporanea pratica 
didattica, basata sull'accumulazione di conoscenze, sull'attenzione per retorica e stile, sulla classificazione di luoghi comuni; la teoria dell'eroe virgiliano come uomo ideale; la conoscenza del poema attraverso la rilettura e la ripetizione. In ogni caso, emerge una nuova attenzione per la parte iliadica, testimoniata anche dal successo in età rinascimentale del tredicesimo libro composto da Maffeo Vegio.

\section{AUTORI}

\section{AMEDEO ALESSANDRO RASCHIERI}

Università degli Studi di Torino

amedeo.raschieri@unito.it 